\title{
Convergence of the self-dual Ginzburg-Landau gradient flow
}

\author{
Sophia Demoulini \\ Centre for Mathematical Sciences, Wilberforce Road, Cambridge, CB3 OWB, England \\ email:sd290@cam.ac.uk
}

\begin{abstract}
We prove convergence of the gradient flow of the Ginzburg-Landau energy functional on a Riemann surface in the self-dual Bogomolny case, in Coulomb gauge. The proof is direct and makes use of the associated nonlinear first order differential operators (the Bogomolny operators). One aim is to illustrate that the Bogomolny structure, which is known to be of great utility in the static elliptic case, can also be used effectively in evolution problems. We also identify the minimizers and minimum value of the energy when the Bogomolny bound is not achieved (below the Bradlow limit).
\end{abstract}

MSC classification: 58J35, 35Q56

\section{Introduction and statement of results}

We consider the self-dual Ginzburg-Landau, or abelian Higgs, energy defined over a two dimensional Riemann surface $\Sigma$ :

$$
\left.\mathcal{V}(\mathbf{A}, \Phi)=\frac{1}{2} \int_{\Sigma} D_{\mathbf{A}} \Phi \wedge * D_{\mathbf{A}} \Phi+F * F+\frac{1}{4}\left(1-|\Phi|^{2}\right)\right)^{2} * 1
$$

Here $\Phi$, the Higgs field, is a section of a complex line bundle $L \rightarrow \Sigma$ with fixed metric $h$, and $D_{\mathbf{A}}=\left(D_{1}, D_{2}\right)=\nabla-i \mathbf{A}$ is the covariant derivative operator defining an $S^{1}$ connection on $L$, with curvature 2 -form $-i F$. We will assume that the degree $N=\operatorname{deg} L$ is a non-negative integer (but everything in this article has an analogous version in the negative case). It is known from [2] that for $|\Sigma|>4 \pi N$ the minimum value of $\mathcal{V}$ is $\pi N$ and is attained on the set of self-dual vortices, which are solutions of the Bogomolny equations (1.5); these form a system of first order partial differential equations, solutions of which are minimizers of $\mathcal{V}$ when they exist. The presence of such a system of first order equations is a special feature of the functional $\mathcal{V}$ which is related to self-duality ([8, 2]). The main aim of this article is to show how it is possible to prove convergence of the corresponding gradient flow quite simply by making use of the Bogomolny (or self-dual) structure in (1.4)-(1.5); it is also proved that for $|\Sigma|<4 \pi N$ the minimum value of $\mathcal{V}$ is $\pi N+\frac{l^{2}}{2|\Sigma|}$ where $l=2 \pi N-\frac{|\Sigma|}{2}$, and this is achieved by taking $\Phi \equiv 0$ and $\mathbf{A}$ to be a constant curvature connection.

A similar proof of convergence for the $S U(2)$ Yang-Mills-Higgs functional on $\mathbb{R}^{3}$ was carried out in [7]; this is closely related to the case $|\Sigma|>4 \pi N$ in this paper. The crucial structural features exploited there for Yang-Mills-Higgs also hold for the Ginzburg-Landau flow in the case $|\Sigma|>4 \pi N$ : by (3.18) the first order Bogomolny operators (3.16)-(3.17) converge to zero, while (3.30) then implies the convergence of $(\mathbf{A}, \Phi)$ as $t \rightarrow+\infty$. The case $|\Sigma|<4 \pi N$ is different: indeed this latter inequality is an obstruction to the existence of solutions to the Bogomolny equations 
([2]), and so the first stage of the argument just outlined necessarily fails. We give, however, an alternative set of quantities in (3.45), whose large time behaviour can be analyzed, and which serve as an effective alternative to the Bogomolny operators in this case. Compared to the adiabatic approximation method in [4], where convergence of the Ginzburg-Landau flow was proved for the case $\Sigma=\mathbb{R}^{2}$, the proof here is certainly more efficient although it makes less contact with the physics of vortices. It is also possible to prove convergence by the very general method based on the Lojasiewicz inequality in [11, as has been done in [6]. In comparison with these previous results, the present method does provide the additional information that convergence is at an exponential rate. Furthermore in view of the importance of the Bogomolny self-dual structure in the elliptic case, it seems worthwhile to illustrate its utility in the parabolic context, as was done also in [3] for vortex dynamics in a conservative (Hamiltonian) context.

Introducing conformal coordinates $\left\{x^{j}\right\}_{j=1}^{2}$, in which the metric takes the form

$$
g=g_{j k} d x^{j} d x^{k}=e^{2 \rho}\left(\left(d x^{1}\right)^{2}+\left(d x^{2}\right)^{2}\right)
$$

with associated area form $d \mu_{g}=e^{2 \rho} d x^{1} \wedge d x^{2}$, the energy functional $\mathcal{V}$ is given by

$$
\left.\mathcal{V}(\mathbf{A}, \Phi)=\frac{1}{2} \int_{\Sigma}\left(\left|D_{\mathbf{A}} \Phi\right|^{2} e^{-2 \rho}+B^{2}+\frac{1}{4}\left(1-|\Phi|^{2}\right)\right)^{2}\right) d \mu_{g}
$$

If we fix a smooth connection $\nabla$ on $L$ then $D_{\mathbf{A}}$ is determined uniquely by a real 1-form

$$
\mathbf{A}=A_{1} d x^{1}+A_{2} d x^{2} \in \Omega^{1}(\Sigma)
$$

where $A_{i}: \Sigma \rightarrow \mathbb{R}$, according to

$$
D_{\mathbf{A}}:=\nabla-i \mathbf{A}
$$

The curvature, or magnetic field $B$, of a connection $D_{\mathbf{A}}=D_{j} d x^{j}$ is determined by $D_{j} D_{k} \Phi d x^{j} \wedge$ $d x^{k}=-i F \Phi=-i B \Phi d \mu_{g}$. Its integral is a topological invariant of $L$ :

$$
\int_{\Sigma} B d \mu_{g}=2 \pi N
$$

with $N=\operatorname{deg} L$. We will choose $\nabla$ to have constant curvature $b$; this value is then fixed topologically as $b=2 \pi N /|\Sigma|$, where $|\Sigma|=\int_{\Sigma} d \mu_{g}$ is the area of $\Sigma$. It follows that

$$
B=b+* d \mathbf{A} \equiv b+e^{-2 \rho}\left(\partial_{1} A_{2}-\partial_{2} A_{1}\right) .
$$

\subsection{The Bogomolny equations}

A crucial property of the energy functional (1.1) is that (in appropriate functions spaces in which e.g. integration by parts is valid) it admits a Bogomolny decomposition into a sum of squares of first order terms:

$$
\mathcal{V}(\mathbf{A}, \Phi)=\frac{1}{2} \int_{\Sigma}\left(4\left|\bar{\partial}_{\mathbf{A}} \Phi\right|^{2} e^{-2 \rho}+\left(B-\frac{1}{2}\left(1-|\Phi|^{2}\right)\right)^{2}\right) d \mu_{g}+\pi N
$$

where as above $N=\operatorname{deg} L$ and

$$
\bar{\partial}_{\mathbf{A}} \Phi=\frac{1}{2}\left(D_{1}+i D_{2}\right) \Phi
$$


If the following first order equations, called the Bogomolny equations,

$$
\begin{gathered}
\bar{\partial}_{\mathbf{A}} \Phi=0, \\
B-\frac{1}{2}\left(1-|\Phi|^{2}\right)=0
\end{gathered}
$$

have solutions in a given class, they will automatically minimize $\mathcal{V}$ within that class (this theory and conditions under which solutions exist - is developed for the plane and for surfaces in [8, 10, 2] ).

Our main theorem concerns the large time behaviour of global weak (Sobolev) solutions to 1.6 in Coulomb gauge (the existence and uniqueness of which is shown in section 2). We show that as $t \rightarrow \infty$ this solution converges exponentially fast to a minimizer of (1.1) a solution of the Bogomolny equations when $|\Sigma|>4 \pi N$. We make the assumption of small (close to equilibrium) initial energy.

We will make use of the Bogomolny equations to derive the asymptotic convergence of weak solutions to the gradient flow equations in section 3. In fact, the convergence of such solutions to minimizers of the energy provides an independent proof of the existence of minimizers and solutions to the Bogomolny equations in the case when $|\Sigma|>4 \pi N$.

\subsection{The time-dependent equations and statement of the main theorem}

Associated with the energy functional (1.1) are the Ginzburg-Landau gradient flow equations in the variables $(\mathbb{A}, \Phi) \equiv\left(A_{0}, \mathbf{A}, \Phi\right) \equiv\left(A_{0}, A_{1}, A_{2}, \Phi\right)$ on $[0, \infty) \times \Sigma$ :

$$
\left(\frac{d}{d t}, D_{0}\right)(\mathbf{A}, \Phi)=-\mathcal{V}(\mathbf{A}, \Phi)
$$

and equivalently,

$$
\begin{gathered}
\frac{\partial A_{1}}{\partial t}-\frac{\partial A_{0}}{\partial x^{1}}=-\frac{\partial B}{\partial x^{2}}+\left\langle i \Phi, D_{1} \Phi\right\rangle, \\
\frac{\partial A_{2}}{\partial t}-\frac{\partial A_{0}}{\partial x^{2}}=+\frac{\partial B}{\partial x^{1}}+\left\langle i \Phi, D_{2} \Phi\right\rangle \\
D_{0} \Phi-\Delta_{\mathbf{A}} \Phi-\frac{1}{2}\left(1-|\Phi|^{2}\right) \Phi=0
\end{gathered}
$$

where the covariant Laplacian $\Delta_{\mathbf{A}}$ is defined as

$$
\Delta_{\mathbf{A}}=e^{-2 \rho}\left(D_{1}^{2}+D_{2}^{2}\right)
$$

This is the fully gauge invariant version of the Ginzburg-Landau gradient flow. The Higgs field $\Phi$, now depending on time $t \geq 0$, is a section of a complex line bundle $\mathbb{L} \equiv[0, \infty) \times L \rightarrow[0, \infty) \times \Sigma$ on which there is a connection

$$
\mathbb{A}=\left(A_{0}, A_{1}, A_{2}\right)=\left(A_{0}, \mathbf{A}\right)
$$

with associated covariant derivative $\mathbb{D}=\left(D_{0}, D_{1}, D_{2}\right)=\left(D_{0}, D_{\mathbf{A}}\right)$, where $D_{0}=\frac{\partial}{\partial t}-i A_{0}$ is the time component and $D_{\mathbf{A}}=\left(D_{1}, D_{2}\right)$ is the spatial component as in (1.2) with $A_{i}$ now depending on time $t \geq 0$ as well as $x \in \Sigma$. The dependent variables are thus the Higgs field $\Phi$ and a real 1 -form $A_{0} d t+A_{j} d x^{j}$ on $[0, \infty) \times \Sigma$. The system is invariant under the infinite dimensional group of gauge transformations: if $\chi(t, x)$ is a smooth function then $\left(A_{0}, A_{1}, A_{2}, \Phi\right)$ is a smooth solution of (1.6) if and only if $\left(A_{0}+\partial_{t} \chi, A_{1}+\partial_{1} \chi, A_{2}+\partial_{2} \chi, e^{i \chi} \Phi\right)$ is a smooth solution of (1.6). This means it 
is possible to place further conditions on the solution, and this is necessary to obtain uniqueness. We impose the Coulomb gauge condition:

$$
\operatorname{div} \mathbf{A}=e^{-2 \rho}\left(\partial_{1} A_{1}+\partial_{2} A_{2}\right)=0 ;
$$

a solution, or set of initial data, is said to be in Coulomb gauge when this condition holds for all relevant $t$. In addition, we will require that

$$
\int_{\Sigma} A_{0} d \mu_{g}=0
$$

at all relevant $t$ : this may be achieved by applying to the solution $\left(A_{0}, A_{1}, A_{2}, \Phi\right)$ at each $(t, x)$ the gauge transformation $\chi(t)=-t|\Sigma|^{-1} \int_{\Sigma} A_{0} d \mu_{g}$.

Initial data and Coulomb gauge: We specify $(\mathbf{A}, \Phi)=\left(A_{1}, A_{2}, \Phi\right)$ at $t=0$ which in Coulomb gauge also determines $A_{0}$ initially. To see this, we eliminate $A_{0}$ from the full (gauge invariant) equations (1.6) by taking the divergence in the equation for $\mathbf{A}$ under the condition (1.7). This leads to the equation for $A_{0}$ in Coulomb gauge,

$$
-\Delta A_{0}=\operatorname{div}\left\langle i \Phi, D_{\mathbf{A}} \Phi\right\rangle \text {. }
$$

Lemma 1.1 For each fixed $t \geq 0$ there is a smooth function $(\mathbf{A}, \Phi) \mapsto \alpha(\mathbf{A}, \Phi)$ from $H^{1}\left(L \oplus \Omega^{1}\right) \rightarrow$ $H^{1, q}$, for any $1<q<2$, which is the unique solution of (1.9) satisfying (1.8).

Proof Because $\Phi \in H^{1}$ then for all $p<\infty, \Phi \in L^{p}, D_{\mathbf{A}} \Phi \in L^{2}$ and so $\operatorname{div}\left\langle i \Phi, D_{\mathbf{A}} \Phi\right\rangle \in H^{-1, q}$ for all $q<2$. It follows that (1.9) has a unique solution $A_{0}=\alpha(\mathbf{A}, \Phi)$ verifying (1.8) as described in the lemma.

Remark 1.2 Furthermore, if $\Phi$ satisfies (1.6) then for t a.e. $-\Delta A_{0}=\operatorname{div}\left\langle i \Phi, D_{\mathbf{A}} \Phi\right\rangle=\left\langle i \Phi, D_{0} \Phi\right\rangle$, and so by standard elliptic theory,

$$
\left\|A_{0}\right\|_{H^{2, p}} \leq c_{s}\left\|\left\langle i \Phi, D_{0} \Phi\right\rangle\right\|_{L^{p}},
$$

where finiteness of the right hand side will be justified in section 3.1. By Sobolev imbeddings, here applied for $1<p<2$ at each time $t \geq 0$,

$$
\left\|A_{0}\right\|_{H^{2, p}} \leq C_{1}\|\Phi\|_{L^{\frac{2 p}{2-p}}}\left\|D_{0} \Phi\right\|_{L^{2}} \quad \text { and so } \quad\left\|d A_{0}\right\|_{L^{2}} \leq C_{2}\|\Phi\|_{H_{\mathbf{A}}^{1}}\left\|D_{0} \Phi\right\|_{L^{2}} .
$$

As a particular consequence, $A_{0}$ is initially determined through the initial data for $(\mathbf{A}, \Phi)$. In addition, the estimates (1.10) will be used below to derive estimates for $D_{0} \Phi$.

Explicitly applying the gauge conditions (1.7) and (1.8) and substituting $A_{0}=\alpha(\mathbf{A}$, $\Phi)$, the system (1.6) becomes the following nonlocal parabolic system:

$$
\begin{aligned}
\dot{\mathbf{A}} & =\Delta \mathbf{A}+d \alpha+\left\langle i \Phi, D_{\mathbf{A}} \Phi\right\rangle \\
\dot{\Phi} & =\Delta \Phi-2 i e^{-2 \rho} \sum_{j} A_{j} \partial_{j} \Phi-e^{-2 \rho}|\mathbf{A}|^{2} \Phi+i \alpha \Phi+\frac{1}{2}\left(1-|\Phi|^{2}\right) \Phi
\end{aligned}
$$

with initial data specified for $(\mathbf{A}, \Phi)$. We discuss existence of this system in section 2 and we will need the following spaces. 
Function spaces: We will work with spaces of functions $H^{s, p}(\Sigma)$ which may be defined either (i) by using a partition of unity to reduce to the Euclidean case (the standard spaces $H^{s, p}\left(\mathbb{R}^{2}\right)$ ), or (ii) by appealing to the spectral theory for $-\Delta$ to define a functional calculus and then introducing the norm as

$$
\|f\|_{H^{s, p}}=\left\|(1-\Delta)^{\frac{s}{2}} f\right\|_{L^{p}} .
$$

Let $f_{j}$ be an orthonormal basis of eigenfunctions, $-\Delta f_{j}=\lambda_{j} f_{j}$ with $0 \leq \lambda_{1} \leq \lambda_{2} \leq \ldots$, then for $p=2$ an equivalent norm on $H^{s}=H^{s, 2}$ is

$$
\|f\|_{H^{s}}^{2}=\sum_{j}\left(1+\lambda_{j}\right)^{s}\left|c_{j}\right|^{2}, \quad \text { where } f=\sum c_{j} f_{j} .
$$

These definitions extend to sections of vector bundles $L$, in which case we write $H^{s}(L)$ for the corresponding spaces defined with respect to the connection $\nabla$ and its corresponding Laplacian $e^{-2 \rho}\left(\nabla_{1}^{2}+\nabla_{2}^{2}\right)$; for $s=1$ an equivalent norm is

$$
\|\Phi\|_{H^{1}(L)}^{2}=\int_{\Sigma}\left(|\Phi|^{2}+|\nabla \Phi|^{2}\right) d \mu_{g}
$$

In the above integral the inner products are the standard ones induced from $h$ and $g$. The definitions also extend to 1 -forms using the Hodge Laplacian $\Delta=-(d \delta+\delta d)$, (with the sign chosen so that it is non-positive). In conformal coordinates:

$$
\begin{aligned}
(\Delta \mathbf{A})_{i} & =\partial_{i}\left(e^{-2 \rho}\left(\partial_{1} A_{1}+\partial_{2} A_{2}\right)\right)-\epsilon_{i j} \partial_{j}\left(e^{-2 \rho}\left(\partial_{1} A_{2}-\partial_{2} A_{1}\right)\right) \\
& =-\epsilon_{i j} \partial_{j} B
\end{aligned}
$$

when the Coulomb gauge (1.7) holds. The corresponding norms are written $H^{s}\left(\Omega^{1}\right)$ where $\Omega^{1}$ is the space of one-forms on $\Sigma$ with coefficients in $H^{s}$.

The main theorem

For $H^{1}$ data we derive existence of a solution also in $H^{1}$ in the following section in the Coulomb gauge. The main result concerns the time asymptotic behaviour of these solutions which are shown to be exponentially converging to an energy minimizer. The minimum energy depends on whether the size of $\Sigma$ is large enough to support the existence of vortices ([2, 10]):

$$
\mathcal{V}_{\text {min }}=\left\{\begin{array}{cc}
\pi N & \text { if } \Sigma>4 \pi N \\
\pi N+\frac{(4 \pi N-|\Sigma|)^{2}}{8|\Sigma|} & \text { if } \Sigma \leq 4 \pi N .
\end{array}\right\}
$$

Let

$$
\mathcal{V}_{0}=\mathcal{V}(\mathbf{A}(0), \Phi(0))
$$

be the initial energy. The following is the main theorem

Theorem 1.3 (Main Theorem) Given finite energy initial data $(\mathbf{A}(0), \Phi(0)) \in H^{1}\left(\Omega^{1} \oplus L\right)$ satisfying the Coulomb gauge condition (1.7) and such that $\epsilon_{0}$ with

$$
0 \leq \mathcal{V}_{0}-\mathcal{V}_{\min }=\epsilon_{0}
$$

is sufficiently small there exists a unique global solution $\left(A_{0}, \mathbf{A}, \Phi\right)$ with $\left.A_{0} \in C([0, \infty)) ; H^{1, p}(\Sigma)\right)$ for any $p<2$, and $(\mathbf{A}, \Phi) \in C\left([0, \infty) ; H^{1}\left(\Omega^{1} \oplus L\right)\right)$ of (1.6), satisfying (1.7) and (1.8) with the following large time behaviour: 
if $|\Sigma|>4 \pi N$ then $(\mathbf{A}, \Phi) \stackrel{t \rightarrow \infty}{\longrightarrow}\left(\mathbf{A}_{\infty}, \Phi_{\infty}\right)$ in $L^{2}\left(\Omega^{1} \oplus L\right)$ where $\left(\mathbf{A}_{\infty}, \Phi_{\infty}\right)$ is a solution of the minimum energy static (Bogomolny) equations and where $\Phi_{\infty}$ has precisely $N$ zeros (vortices) on $\Sigma$. Also $A_{0}(t) \rightarrow 0$ strongly in $L^{q}$ for all $q<\infty$. The minimum value of the energy in $H^{1}\left(L \oplus \Omega^{1}\right)$ is attained and is $\pi N$.

if $|\Sigma|<4 \pi N$ then $(\mathbf{A}, \Phi) \stackrel{L^{2}}{\rightarrow}\left(\mathbf{A}_{\infty}, 0\right)$ where $D=\nabla-i \mathbf{A}_{\infty}$ is a constant curvature connection at which the minimum value of the energy in $H^{1}\left(L \oplus \Omega^{1}\right)$ is achieved and equals

$$
\mathcal{V}\left(\mathbf{A}_{\infty}, 0\right)=\min _{H^{1}\left(L \oplus \Omega^{1}\right)} \mathcal{V}=\pi N+\frac{(4 \pi N-|\Sigma|)^{2}}{8|\Sigma|}
$$

Convergence is at an exponential rate, namely, $\exists c, \delta>0$ depending only the initial data such that

$$
\left\|(\mathbf{A}, \Phi)-\left(\mathbf{A}_{\infty}, \Phi_{\infty}\right)\right\|_{L^{2}} \leq c e^{-\delta t}, \quad \text { respectively, } \quad\left\|(\mathbf{A}, \Phi)-\left(\mathbf{A}_{\infty}, 0\right)\right\|_{L^{2}} \leq c e^{-\delta t} .
$$

Global existence for (1.6) is established in the following section, making use of standard (semigroup) techniques (theorem 2.1). The proof of asymptotic convergence, which is the main content of theorem 1.3, is then given in section 3 .

Remark 1.4 Convergence in stronger $H^{s}$ norms for $s<1$ can be deduced by interpolation between $L^{2}$ and $H^{1}$ from (1.14), using convergence in $L^{2}$ ("low" norm) and boundedness in $H^{1}$ (or "high" norm) guaranteed the energy non-increase. Furthermore, when boundedness can be proved in higher norms than $H^{1}$ then interpolation would lead to convergence in $H^{s}$ for higher s by standard parabolic theory.

Remark 1.5 The case

$$
|\Sigma|=4 \pi N
$$

is degenerate (as becomes clear from reading the proof) and it is conceivable that exponential convergence may not occur, even if the initial energy is close to the minimum energy.

Remark 1.6 The condition that the initial energy be close to the minimum energy can be relaxed in situations when there are no non-minimal critical points ([8, chapter 3]). The energy is strictly decreasing (as it is a gradient flow) and so it must decrease to the energy of a critical point. If nonminimal critical points do not exist then the energy must decrease to its minimum value, in which case it approaches the minimum energy, and then the conclusion of the theorem holds regarding exponential convergence. This is not discussed here further.

\section{Existence theorem}

There are different ways to obtain a global existence theorem for these equations, for example using maximum principles (as for the case of the same equations on $\mathbb{R}^{2}$ in [4]) or energy methods as done here, using semi-group techniques explained in e.g. [12] and elsewhere. To use the energy norm method, we first reduce our system in Coulomb gauge to a system for $(\mathbf{A}, \Phi)$ given in (1.11); then we show that for $H^{1}$ data $(\mathbf{A}(0), \Phi(0))$ there is a unique global weak solution of those equations, also in $H^{1}$, varying continuously in time. 
Theorem 2.1 (Existence in Coulomb gauge) Given initial data $(\mathbf{A}(0), \Phi(0)) \in H^{1}\left(\Omega^{1} \oplus L\right)$ (which implies finite initial energy $\left.\mathcal{V}(\mathbf{A}(0), \Phi(0))=\mathcal{V}_{0}<\infty\right)$, there exists a unique mild solution of (1.11) with regularity $(\mathbf{A}, \Phi) \in C\left([0, \infty) ; H^{1}\left(\Omega^{1} \oplus L\right)\right.$, which satisfies

$$
\mathcal{V}((\mathbf{A}, \Phi)(t)) \leq \mathcal{V}((\mathbf{A}, \Phi)(0))
$$

for all $t \geq 0$. For smooth initial data the solution is smooth, and for $H^{1}\left(\Omega^{1} \oplus L\right)$ initial data the solution is the limit in $C_{l o c}\left([0, \infty) ; H^{1}\left(\Omega^{1} \oplus L\right)\right.$ of smooth solutions.

Proof Writing $\mathcal{U}=(\mathbf{A}, \Phi)$ and $\mathcal{L U}=(\Delta \mathbf{A}, \Delta \Phi)$ the system (1.11) is of the form $\dot{\mathcal{U}}=\mathcal{L U}+\mathcal{F}(\mathcal{U})$, which can be treated as an abstract evolution equation in the space $X=H^{1}\left(\Omega^{1} \oplus L\right)$. We introduce also the auxiliary space $Y=H^{-\frac{1}{2}}\left(\Omega^{1} \oplus L\right)$, and make use of the following facts:

- $e^{t \mathcal{L}}$ is a strongly continuous semi-group of contractions on $X$ for $t \geq 0$,

- $e^{t \mathcal{L}}: Y \rightarrow X$ for $t>0$, with $\left\|e^{t L}\right\|_{Y \rightarrow X} \leq C t^{-3 / 4}$ for $0<t \leq 1$,

- $\mathcal{F}: X \rightarrow Y$ is a smooth function satisfying $\left\|\mathcal{F}\left(\mathcal{U}_{1}\right)-\mathcal{F}\left(\mathcal{U}_{2}\right)\right\|_{Y} \leq K(R)\left\|\mathcal{U}_{1}-\mathcal{U}_{2}\right\|_{X}$ for $\left\|\mathcal{U}_{j}\right\|_{X} \leq R$.

The first of these is a standard property of the heat equation. The second can be derived using the spectral representation introduced above:

$$
e^{t \Delta} \sum c_{j} f_{j}=\sum e^{-t \lambda_{j}} c_{j} f_{j}
$$

which implies that

$$
\left\|e^{t \Delta} f\right\|_{H^{s}}^{2}=\sum e^{-2 t \lambda_{j}}\left(1+\lambda_{j}\right)^{s}\left|c_{j}\right|^{2} \leq C_{s, r}(t)^{2}\|f\|_{H^{r}}^{2}
$$

with $C_{s, r}(t)=\sup _{x \geq 0} \frac{(1+x)^{\frac{s}{2}} e^{-t x}}{(1+x)^{\frac{r}{2}}} \sim c t^{-\frac{s-r}{2}}$ as $t \rightarrow 0$. The third assertion follows by examining the various terms which constitute $\mathcal{F}$ and applying appropriate embeddings for the $H^{s}$ spaces, in particular $H^{\frac{1}{2}} \subset L^{4}$ and $L^{\frac{4}{3}} \subset H^{-\frac{1}{2}}$ (continuous embeddings). For example, the current term $\langle i \Phi, \nabla \Phi\rangle$ in the $\Phi$ equation arises as a continuous bilinear map $H^{1} \times L^{2} \rightarrow L^{\frac{4}{3}} \subset H^{-\frac{1}{2}}$ bounded by $|\langle i \Phi, \nabla \Phi\rangle|_{L^{\frac{4}{3}}} \leq c\|\Phi\|_{L^{4}}\|\nabla \Phi\|_{L^{2}}$. The other terms in $\mathcal{F}$ are treated similarly (using lemma 1.1 to handle the $\alpha$ terms).

These three properties imply (see [12, §15.1] that the integral operator $\mathcal{U} \rightarrow \int_{0}^{t} e^{(t-s) \mathcal{L}} \mathcal{F}(\mathcal{U}(s)) d s$ is a contraction on $C([0, T] ; X)$ for sufficiently small $T$, and in fact $T$ can be taken to be a positive non-increasing function of $\|\mathcal{U}(0)\|_{X}$. This implies that there is a unique local solution $\mathcal{U} \in C([0, T] ; X)$, and also that, given two sets of initial data $\mathcal{U}_{1}(0), \mathcal{U}_{2}(0)$ in $X$, the well-posedness estimate

$$
\max _{0 \leq t \leq T}\left\|\mathcal{U}_{1}(t)-\mathcal{U}_{2}(t)\right\|_{X} \leq c\left\|\mathcal{U}_{1}(0)-\mathcal{U}_{2}(0)\right\|_{X}
$$

holds for some $c>0$, for $T$ sufficiently small (again depending only on $\left\|\mathcal{U}_{j}(0)\right\|_{X}$.) From this it can be deduced that for $H^{2}$ initial data the solution remains in $H^{2}$, and in fact for $H^{s}$ initial data the solution remains in $H^{s}$ for all $s \geq 2$. Thus the $H^{1}$ solutions can be approximated by regular solutions, and obey the energy non-increase : $\mathcal{V}((\mathbf{A}, \Phi)(t)) \leq \mathcal{V}((\mathbf{A}, \Phi)(s))$ for $t \geq s$. 
As a consequence the $H^{1}$ norm is globally bounded and there exists a unique global solution $\mathcal{U} \in C([0, \infty) ; X)$ of the corresponding integral equation

$$
\mathcal{U}(t)=e^{t \mathcal{L}} \mathcal{U}(0)+\int_{0}^{t} e^{(t-s) \mathcal{L}} \mathcal{F}(\mathcal{U}(s)) d s
$$

with the approximation property asserted.

\section{Asymptotic behaviour and proof of the main theorem}

We continue to use the Coulomb gauge in which $A_{0}$ is eliminated as an independent variable by lemma 1.1. We consider the cases $|\Sigma|>4 \pi N$ and $|\Sigma|<4 \pi N$ separately. In each case we introduce auxiliary variables which are specially tailored to reveal the asymptotic convergence of $(\mathbf{A}, \Phi)$ to a limit $\left(\mathbf{A}_{\infty}, \Phi_{\infty}\right)$ which is characterized differently depending on whether the surface area $|\Sigma|$ is bigger or smaller than $4 \pi N$. In the following proofs we shall make use of the norm

$$
\|\eta\|_{H_{\mathbf{A}}^{1}}^{2}=\int_{\Sigma}\left(|\eta|^{2}+\left|D_{\mathbf{A}} \eta\right|^{2}\right) d \mu_{g}
$$

defined with respect to the time dependent connection $D_{\mathbf{A}}=\nabla-i \mathbf{A}$.

\subsection{Proof in the case $|\Sigma|>4 \pi N$}

It is useful to introduce the Bogomolny variables $v=v(\mathbf{A}, \Phi)$ and $\eta=\eta(\mathbf{A}, \Phi)$, defined as

$$
\begin{aligned}
\eta & =\bar{\partial}_{\mathbf{A}} \Phi \\
v & =B-\frac{1}{2}\left(1-|\Phi|^{2}\right)
\end{aligned}
$$

where, as above, $\bar{\partial}_{\mathbf{A}}=\frac{1}{2}\left(D_{1}+i D_{2}\right)$. In terms of these variables the energy decomposes:

$$
\mathcal{V}=\frac{1}{2} \int_{\Sigma}\left(4|\eta|^{2} e^{-2 \rho}+v^{2}\right) d \mu_{g}+\pi N
$$

as was seen above in (1.4). The evolution of these variables is according to

$$
\begin{aligned}
& D_{0} \eta-4 \bar{\partial}_{\mathbf{A}}\left(e^{-2 \rho} \partial_{\mathbf{A}} \eta\right)+|\Phi|^{2} \eta=-v \eta \\
& \left(\frac{\partial}{\partial t}-\Delta+|\Phi|^{2}\right) v=-4|\eta|^{2} .
\end{aligned}
$$

These equations can be obtained from (1.6) by first applying the operator $\bar{\partial}_{\mathbf{A}}$ to the equation for $\Phi$, using the identities (in conformal co-ordinates)

$$
4 \partial_{\mathbf{A}} \bar{\partial}_{\mathbf{A}}=D_{1}^{2}+D_{2}^{2}+e^{2 \rho} B, \quad \Delta_{\mathbf{A}}=e^{-2 \rho}\left(D_{1}^{2}+D_{2}^{2}\right)
$$

and

$$
\bar{\partial}_{\mathbf{A}} \Delta_{\mathbf{A}}-4 \bar{\partial}_{\mathbf{A}}\left(e^{-2 \rho} \partial_{\mathbf{A}}\right) \bar{\partial}_{\mathbf{A}}=-(\bar{\partial} B)-B \bar{\partial}_{\mathbf{A}}
$$


and

$$
\left[\bar{\partial}_{\mathbf{A}}, D_{0}\right]=\frac{i}{2}\left(\dot{A}_{1}+i \dot{A}_{2}\right)-i \bar{\partial} A_{0}
$$

(where $\bar{\partial} A_{0}=\left(\bar{\partial} A_{0}\right)+A_{0} \bar{\partial}$ ) and finally using the equation for $\mathbf{A}$ in the last commutator. (To derive the final commutator use also that the background connection $\nabla$ is fixed independent of $t$, i.e., $\left[\frac{\partial}{\partial t}, \nabla\right]=0$.)

We will show (using lemma 3.2) that

$$
(\eta, v) \longrightarrow(0,0) \text { as } t \rightarrow \infty
$$

(in specified spaces) and then deduce via lemma 3.4 the convergence of $(\mathbf{A}, \Phi)$ as stated in the main theorem. For this consider two energy-type quadratic forms, respectively associated to each of the equations in 3.18 (at each time $t>0$ ) defined as

$$
\begin{aligned}
Q_{\mathbf{A}, \Phi}(\eta) & =\int_{\Sigma}\left(4 e^{-4 \rho}\left|\partial_{\mathbf{A}} \eta\right|^{2}+e^{-2 \rho}|\Phi|^{2}|\eta|^{2}\right) d \mu_{g} \\
Q_{\Phi}(v) & =\int_{\Sigma}\left(e^{-2 \rho}\left(\left|D_{1} v\right|^{2}+\left|D_{2} v\right|^{2}\right)+|\Phi|^{2} v^{2}\right) d \mu_{g} .
\end{aligned}
$$

Remark 3.1 It is shown in [3][lemma 3.2.2] that these quadratic forms are coercive in $H_{\mathbf{A}}^{1}$ and $H^{1}$ respectively, provided that $\|\Phi\|_{L^{2}} \geq m>0$ : to be precise, under this assumption on $\Phi$, there exists $\gamma=\gamma(m, M)>0$ (where recall that $\mathcal{V} \leq M<\infty$ and $M=\pi N+1$ ), such that

$$
Q_{\mathbf{A}, \Phi}(\eta) \geq \gamma\|\eta\|_{H_{\mathbf{A}}^{1}}^{2} \quad \text { and } \quad Q_{\Phi}(v) \geq \gamma\|v\|_{H^{1}}^{2} .
$$

That the assumption on $\|\Phi\|_{L^{2}}$ is a valid one can be shown as follows: by the Bogomolny decomposition of the functional $\mathcal{V}(1.4)$ and the topological invariant (1.3),

$$
\int v=2 \pi N-\frac{|\Sigma|}{2}+\frac{\|\Phi\|_{L^{2}}^{2}}{2}
$$

from which it follows that,

$$
\begin{aligned}
\|\Phi\|_{L^{2}}^{2} & =|\Sigma|-4 \pi N+2 \int v \\
& \geq|\Sigma|-4 \pi N-2\left(2 \epsilon_{0}\right)^{\frac{1}{2}}|\Sigma|^{\frac{1}{2}} \\
& >0
\end{aligned}
$$

for $\epsilon_{0}$ sufficiently small.

In terms of the quadratic forms $Q_{\mathbf{A}, \Phi}, Q_{\Phi}$ the variables $\eta, v$ satisfy the following inequalities:

Lemma 3.2 Let $(\mathbf{A}, \Phi)$ be as in theorem 2.1 and define $(\eta, v)$ as in (3.16)-(3.17). Then for any time $T>0$, the following identities hold

$$
\begin{aligned}
& e^{2 \delta T}\|\eta(T)\|_{L^{2}}^{2}+2 \int_{0}^{T} e^{2 \delta t}\left(Q_{\mathbf{A}, \Phi}(\eta)-\delta\|\eta\|_{L^{2}}^{2}\right) d t \leq\|\eta(0)\|_{L^{2}}^{2}-2 \int_{0}^{T} \int_{\Sigma} e^{2 \delta t} v|\eta|^{2} e^{-2 \rho} d \mu_{g} d t \\
& e^{2 \delta T}\|v(T)\|_{L^{2}}^{2}+2 \int_{0}^{T} e^{2 \delta t}\left(Q_{\Phi}(v)-\delta\|v\|_{L^{2}}^{2}\right) d t \leq\|v(0)\|_{L^{2}}^{2}-8 \int_{0}^{T} \int_{\Sigma} e^{2 \delta t} v|\eta|^{2} e^{-2 \rho} d \mu_{g} d t .
\end{aligned}
$$


Proof For smooth solutions multiply 3.18 respectively by $\eta$ and $v$ and integrate with respect to $e^{2 \delta t} e^{-2 \rho} d \mu_{g} d t$ over $[0, T] \times \Sigma$. For more general finite energy solutions use the approximation property in theorem 2.1 .

The above lemma implies the exponential decay of $\eta, v$ under the conditions on $\Phi$ for co-ercivity and for initial energy sufficiently close to its minimum.

Corollary 3.3 (Exponential decay) For all $\delta \in\left(0, \frac{\gamma}{2}\right)$ and $0<\epsilon_{0}$ with $\sqrt{2 \epsilon_{0}} \leq \frac{\delta}{8 C_{s}^{2}}$, if $\mathcal{V}(A, \Phi)-$ $\pi N \leq \epsilon_{0}$, then

$$
\sup _{t>0} e^{2 \delta t}\left(\|\eta(t)\|_{L^{2}}^{2}+\|v(t)\|_{L^{2}}^{2}\right)+\delta \int_{0}^{\infty} e^{2 \delta t}\left(\|\eta\|_{H_{\mathbf{A}}^{1}}^{2}+\|v\|_{H^{1}}^{2}\right) d t \leq 2\left(\|\eta(0)\|_{L^{2}}^{2}+\|v(0)\|_{L^{2}}^{2}\right) .
$$

Proof Recombining the terms in (3.23) we have the following bounds

$$
\begin{aligned}
2 \int_{0}^{T} e^{2 \delta t}\left(Q_{\mathbf{A}, \Phi}(\eta)-\delta\|\eta\|_{L^{2}}^{2}\right) d t & +2 \int_{0}^{T} \int_{\Sigma} e^{2 \delta t} v|\eta|^{2} e^{-2 \rho} d \mu_{g} d t \\
& \geq 2 \int_{0}^{T} e^{2 \delta t}(\gamma-\delta)\|\eta\|_{H_{\mathbf{A}}^{1}}^{2} d t-2 \sup _{t}\|v\|_{L^{2}} \int_{0}^{T} e^{2 \delta t}\|\eta\|_{L^{4}}^{2} d t \\
& \geq \int_{0}^{T} e^{2 \delta t}\left(2 \gamma-2 \delta-4 C_{s}^{2} \sup _{t}\|v\|_{L^{2}}\right)\|\eta\|_{H_{\mathbf{A}}^{1}}^{2} d t
\end{aligned}
$$

as there exists $C_{s}>0$ such that $\|\eta\|_{L^{4}} \leq C_{s}\|\eta\|_{H_{\mathrm{A}}^{1}}$ by the Sobolev inequality. (Covariant Sobolev spaces and extensions of standard inequalities in these were discussed in the appendix in [3]). By the energy non-increase,

$$
\sup _{[0, T]}\|v\|_{L^{2}} \leq \sqrt{2(\mathcal{V}-\pi N)} \leq \sqrt{2\left(\mathcal{V}_{0}-\pi N\right)}=\sqrt{2 \epsilon_{0}}
$$

and so for $\sqrt{2 \epsilon_{0}} \leq \frac{2 \gamma-3 \delta}{4 C_{s}^{2}}$ (which is implied by the assumption on $\epsilon_{0}$ ), the final term on the right hand side of (3.26) is bounded below as

$$
\geq \delta \int_{0}^{T} e^{2 \delta t}\|\eta\|_{H_{\mathbf{A}}^{1}}^{2} d t
$$

Hence from (3.23)

$$
\sup _{t \in[0, T]} e^{2 \delta t}\|\eta(t)\|_{L^{2}}^{2}+\delta \int_{0}^{T} e^{2 \delta t}\|\eta\|_{H_{\mathbf{A}}^{1}}^{2} d t \leq\|\eta(0)\|_{L^{2}}^{2} .
$$

Using this and (3.27) (together with Holder's inequality and the same Sobolev imbedding as above) we can bound the final term on the right hand side of (3.24):

$$
8 \int_{0}^{T} \int_{\Sigma} e^{2 \delta t} v|\eta|^{2} e^{-2 \rho} d \mu_{g} d t \leq 8 \int_{0}^{T} \int_{\Sigma} e^{2 \delta t}\|v\|_{L^{2}} C_{s}^{2}\|\eta\|_{H_{\mathbf{A}}^{1}}^{2} d t \leq \frac{8 C_{s}^{2} \sqrt{2 \epsilon_{0}}}{\delta}\|\eta(0)\|_{L^{2}}^{2} \leq\|\eta(0)\|_{L^{2}}^{2}
$$

for $\sqrt{2 \epsilon_{0}} \leq \frac{\delta}{8 C_{s}^{2}}$. Using this and (3.28) in (3.46) we have

$$
\delta \int_{0}^{T} e^{2 \delta t}\|v\|_{H^{1}}^{2} d t \leq 2 \int_{0}^{T} e^{2 \delta t}\left(Q_{\Phi}(v)-\delta\|v\|_{L^{2}}^{2}\right) d t \leq\left(\|\eta(0)\|_{L^{2}}^{2}+\|v(0)\|_{L^{2}}^{2}\right)
$$


which proves the corollary.

In terms of $v=B-\frac{1}{2}\left(1-|\Phi|^{2}\right)$ and $\eta=\bar{\partial}_{\mathbf{A}} \Phi=\frac{1}{2}\left(D_{1}+i D_{2}\right) \Phi$, the equations (1.6) read (in gauge invariant form):

$$
\begin{aligned}
& \frac{\partial A_{1}}{\partial t}-\frac{\partial A_{0}}{\partial x^{1}}=-2\langle\Phi, i \eta\rangle-\frac{\partial v}{\partial x^{2}}, \\
& \frac{\partial A_{2}}{\partial t}-\frac{\partial A_{0}}{\partial x^{2}}=-2\langle\Phi, \eta\rangle+\frac{\partial v}{\partial x^{1}}, \\
& D_{0} \Phi=4 e^{-2 \rho} \partial_{\mathbf{A}} \eta-v \Phi .
\end{aligned}
$$

We will now show that the equations (3.30) together with the estimates (3.25) and also (1.10) for $A_{0}$ imply the convergence as $t \rightarrow \infty$ in $L^{2}$ of $t \mapsto(\mathbf{A}, \Phi)(t)$ at an exponential rate.

Lemma 3.4 In terms of $\eta, v$ above,

$$
\|\dot{\mathbf{A}}\|_{L^{2}}+\|\dot{\Phi}\|_{L^{2}} \leq c\left(\|\eta\|_{H_{\mathbf{A}}^{1}}+\|v\|_{H^{1}}\right)
$$

which together with the estimate (3.25) completes the proof of the theorem in the case $|\Sigma|>4 \pi N$.

Proof Unless specified otherwise the generic constants $c, c^{\prime}$ depend only on $\Sigma$.

Firstly,

$$
\int_{\Sigma}\left(1-|\Phi|^{2}\right)^{2}=|\Sigma|-2\|\Phi\|_{L^{2}}^{2}+\|\Phi\|_{L^{4}}^{4} \geq|\Sigma|-2\|\Phi\|_{L^{4}}^{2}|\Sigma|^{\frac{1}{2}}+\|\Phi\|_{L^{4}}^{4} \geq \frac{1}{2}\|\Phi\|_{L^{4}}^{4}-|\Sigma| .
$$

(as $\left.\|\Phi\|_{L^{2}}^{2} \leq\|\Phi\|_{L^{4}}^{2}|\Sigma|^{\frac{1}{2}} \leq \frac{1}{4}\|\Phi\|_{L^{4}}^{4}+|\Sigma|\right)$. Therefore,

$$
\mathcal{V}(\mathbf{A}, \Phi) \geq c\left(\left\|D_{\mathbf{A}} \Phi\right\|_{L^{2}}^{2}+\|\Phi\|_{L^{4}}^{4}\right)-c^{\prime} \geq c\|\Phi\|_{H_{\mathbf{A}}^{1}}^{2}-c^{\prime}
$$

and using the energy non-increase (shown at the end of the proof of the existence theorem 2.1) this implies

$$
\|\Phi\|_{L^{4}}^{4} \leq c\left(\mathcal{V}_{0}, \Sigma\right)
$$

where $\mathcal{V}_{0}$ is the initial energy.

The last equation in (3.30) implies

$$
\begin{aligned}
\left\|D_{0} \Phi\right\|_{L^{2}}^{2} & \leq c\left(\|\eta\|_{H_{\mathbf{A}}^{1}}^{2}+\|v \Phi\|_{L^{2}}^{2}\right) \\
& \leq c\left(\|\eta\|_{H_{\mathbf{A}}^{1}}^{2}+\|\Phi\|_{L^{4}}^{2}\|v\|_{L^{4}}^{2}\right) \\
& \leq c\left(\mathcal{V}_{0}, \Sigma\right)\left(\|\eta\|_{H_{\mathbf{A}}^{1}}^{2}+\|v\|_{H^{1}}^{2}\right)
\end{aligned}
$$

Therefore by (3.25),

$$
\int_{0}^{\infty}\left\|D_{0} \Phi\right\|_{L^{2}}^{2} e^{2 \delta t} d t \leq \infty
$$

which implies the exponential decay of $t \mapsto D_{0} \Phi$. However, as seen in the previous section, the equation for $\Phi$ implies in the Coulomb gauge

$$
-\Delta A_{0}=\left\langle i \Phi, D_{0} \Phi\right\rangle
$$


and then the Calderon-Zygmund inequality, given the condition $\int A_{0} d \mu_{g}=0$, implies

$$
\left\|A_{0}\right\|_{H^{2, p}} \leq c(\Sigma, p)\left\|\left\langle i \Phi, D_{0} \Phi\right\rangle\right\|_{L^{p}}
$$

For $p<2$,

$$
\begin{aligned}
\left\|\left\langle i \Phi, D_{0} \Phi\right\rangle\right\|_{L^{p}} & \leq\|\Phi\|_{L^{2 p}}\left\|D_{0} \Phi\right\|_{L^{2}} \\
& \leq c\left(\mathcal{V}_{0}, \Sigma\right)\left\|D_{0} \Phi\right\|_{L^{2}} .
\end{aligned}
$$

Therefore, together with (1.10)

$$
\left\|A_{0}\right\|_{H^{2, p}}^{2}+\left\|d A_{0}\right\|_{L^{2}}^{2} \leq c\left\|D_{0} \Phi\right\|_{L^{2}}^{2}
$$

Therefore,

$$
\int_{0}^{\infty}\left(\left\|A_{0}\right\|_{H^{2, p}}^{2}+\left\|d A_{0}\right\|_{L^{2}}^{2}\right) e^{2 \delta t} d t \leq \infty
$$

by (3.33). By the equation for $\Phi$ in (3.30) we also have

$$
\begin{aligned}
\left\|\frac{\partial}{\partial t} \Phi\right\|_{L^{2}} & \leq\left\|A_{0} \Phi\right\|_{L^{2}}+c\left(\|\eta\|_{H_{\mathbf{A}}^{1}}+\|v\|_{H^{1}}\right) \\
& \leq c\left(\mathcal{V}_{0}, \Sigma\right)\left(\left\|D_{0} \Phi\right\|_{L^{2}}+\|\eta\|_{H_{\mathbf{A}}^{1}}+\|v\|_{H^{1}}\right)
\end{aligned}
$$

and so

$$
\int_{0}^{\infty}\left\|\frac{\partial}{\partial t} \Phi\right\|_{L^{2}}^{2} e^{2 \delta t} d t \leq \infty
$$

Similarly for A, we obtain

$$
\int_{0}^{\infty}\left\|\frac{\partial}{\partial t} \mathbf{A}\right\|_{L^{2}}^{2} e^{2 \delta t} d t \leq \infty
$$

These estimates immediately imply (3.31) by the equations (3.30) and then by (3.25) we conclude that

$$
\frac{\partial}{\partial t}(\mathbf{A}, \Phi)=f \text { on }[0, \infty) \times \Sigma
$$

where $f \in L^{2}$ and $\int_{0}^{\infty}\|f\|_{L^{2}}(t) e^{2 \delta t} d t<\infty$, and thus

$$
\left(\mathbf{A}_{\infty}, \Phi_{\infty}\right) \equiv \lim _{t \rightarrow \infty}(\mathbf{A}(t), \Phi(t))
$$

exists in $L^{2}$ with exponential rate of convergence.

From these estimates and the above we have that $\Phi \in H^{1}$ and so is in $L^{p}$ for all $p<\infty$ and as $t \rightarrow \infty, \Phi \rightarrow \Phi_{\infty}$ strongly in $L^{2}, D_{\mathbf{A}} \Phi \rightarrow D_{\mathbf{A}_{\infty}} \Phi_{\infty}$ weakly in $L^{2}$, hence $\Phi D_{\mathbf{A}} \Phi \rightarrow \Phi_{\infty} D_{\mathbf{A}_{\infty}} \Phi_{\infty}$ weakly in $L^{1}$; in addition, as $\Phi$ is bounded in $L^{q}$ for any $q<\infty$ (from $\Phi \in H^{1}$ ), the product $\Phi D_{\mathbf{A}} \Phi$ is bounded in $L^{q}$ for every $q<2$. This implies weak convergence in $L^{q}$ for $q<2$ so that $\operatorname{div}\left(i \Phi, D_{\mathbf{A}} \Phi\right)$ converges weakly in $H^{-1, q}$; by Calderon-Zygmund then $A_{0}$ converges to a limit $A_{0 \infty}$ weakly in $H^{1, q}$ for each $q<2$ and so strongly in every $L^{p}$ for $p<\infty$. Clearly by (3.37) this limit is zero.

This completes the proof of the theorem in the case $|\Sigma|>4 \pi N$. 


\subsection{Proof in the case $|\Sigma|<4 \pi N$}

In this case a different approach is needed because the condition $\|\Phi\|_{L^{2}}^{2} \geq m>0$ which held and was used in the previous section is no longer valid (as is obvious from the explicit form of the lower bound in (3.22) ). Indeed, as first observed in [2], in this case there are no solutions to the Bogomolny equations (1.5). To see this fact, integrate the equation $v=0$ over $\Sigma$ to deduce

$$
2 \pi N-\frac{|\Sigma|}{2}+\frac{\|\Phi\|_{L^{2}}^{2}}{2}=0
$$

which is an impossibility when $4 \pi N|>| \Sigma \mid$. We will show instead that the minimizers of $\mathcal{V}$ have $v=\frac{l}{|\Sigma|}=$ constant and $\|\Phi\|_{L^{2}}=0$, i.e. the field $\Phi$ is identically zero and $D_{\mathbf{A}}=\nabla-i \mathbf{A}$ is a constant curvature connection on $L$. Notice that the value of the constant $l$ is fixed as

$$
l=2 \pi N-\frac{|\Sigma|}{2}>0
$$

since $\int_{\Sigma} v d \mu_{g}=l+\frac{1}{2}\|\Phi\|_{L^{2}}^{2}$ by integration of (3.17). Now define $d>0$ by $d^{2}=|\Sigma| / \int_{\Sigma} v^{2} d \mu_{g}$, then

$$
\begin{aligned}
2\left(\int_{\Sigma} v^{2} d \mu_{g}\right)^{\frac{1}{2}}|\Sigma|^{\frac{1}{2}}=d \int_{\Sigma} v^{2} d \mu_{g}+\frac{|\Sigma|}{d} & =2 \int_{\Sigma} v d \mu_{g}+\int_{\Sigma}\left(\sqrt{d} v-\frac{1}{\sqrt{d}}\right)^{2} d \mu_{g} \\
& =2\left(l+\frac{1}{2}\|\Phi\|_{L^{2}}^{2}\right)+\left\|\left(\sqrt{d} v-\frac{1}{\sqrt{d}}\right)\right\|_{L^{2}}^{2} .
\end{aligned}
$$

Referring to (1.4), this implies that the energy $\mathcal{V}$ can be written

$$
\mathcal{V}(\mathbf{A}, \Phi)=2\left\|\bar{\partial}_{\mathbf{A}} \Phi\right\|_{L^{2}}^{2}+\frac{1}{8|\Sigma|}\left[2\left(l+\frac{1}{2}\|\Phi\|_{L^{2}}^{2}\right)+\left\|\left(\sqrt{d} v-\frac{1}{\sqrt{d}}\right)\right\|_{L^{2}}^{2}\right]^{2}+\pi N .
$$

From this it follows, since $l>0$, that $\mathcal{V} \geq \pi N+\frac{l^{2}}{2|\Sigma|}$ and that this lower bound is achieved with $\Phi$ identically zero and $B=\frac{2 \pi N}{|\Sigma|}=$ constant, so that $v=\frac{l}{|\Sigma|}=\frac{1}{d}=$ constant. Thus although the Bogomolny bound $\pi N$ is not itself achieved in the case $4 \pi N>|\Sigma|$ we have identified the greatest lower bound and shown that it is achieved with the "pure magnetic" constant curvature connections:

Lemma 3.5 For $|\Sigma|<4 \pi N$

$$
\min _{H^{1}\left(L \oplus \Omega^{1}\right)} \mathcal{V}=\pi N+\frac{(4 \pi N-|\Sigma|)^{2}}{8|\Sigma|}
$$

and this minimum value is achieved by taking the Higgs field $\Phi$ identically zero and $D$ to be a constant curvature connection.

To analyze the gradient flow in this case it is useful to introduce the variable $y=v-l$ (in place of $v$ ), so that (3.18) are replaced by

$$
\begin{aligned}
& D_{0} \eta-4 \bar{\partial}_{\mathbf{A}}\left(e^{-2 \rho} \partial_{\mathbf{A}} \eta\right)+\left(l+|\Phi|^{2}\right) \eta=-y \eta, \\
& \left(\frac{\partial}{\partial t}-\Delta+|\Phi|^{2}\right) y=-|\Phi|^{2} l-4|\eta|^{2}, \\
& D_{0} \Phi-4 e^{-2 \rho} \partial_{\mathbf{A}} \bar{\partial}_{\mathbf{A}} \Phi+l \Phi=-y \Phi .
\end{aligned}
$$


Corresponding to (3.23) we have the following integral inequalities for solutions of (3.45)

$$
\begin{aligned}
& e^{2 \delta T}\|\eta(T)\|_{L^{2}}^{2}+2 \int_{0}^{T} e^{2 \delta t}\left(Q_{\mathbf{A}, \Phi}(\eta)+(l-\delta)\|\eta\|_{L^{2}}^{2}\right) d t \leq\|\eta(0)\|_{L^{2}}^{2}-2 \int_{0}^{T} \int_{\Sigma} e^{2 \delta t} y|\eta|^{2} e^{-2 \rho} d \mu_{g} d t \\
& e^{2 \delta T}\|y(T)\|_{L^{2}}^{2}+2 \int_{0}^{T} e^{2 \delta t}\left(Q_{\Phi}(y)-\delta\|y\|_{L^{2}}^{2}\right) d t \leq\|y(0)\|_{L^{2}}^{2}-2 \int_{0}^{T} \int_{\Sigma} e^{2 \delta t} y\left(|\Phi|^{2} l+4|\eta|^{2}\right) e^{-2 \rho} d \mu_{g} d t \\
& e^{2 \delta T}\|\Phi(T)\|_{L^{2}}^{2}+2 \int_{0}^{T} e^{2 \delta t}\left(\left\|\bar{\partial}_{\mathbf{A}} \Phi\right\|_{L^{2}}^{2}+(l-\delta)\|\Phi\|_{L^{2}}^{2}\right) d t \leq\|\Phi(0)\|_{L^{2}}^{2}-2 \int_{0}^{T} \int_{\Sigma} e^{2 \delta t} y|\Phi|^{2} e^{-2 \rho} d \mu_{g} d t .
\end{aligned}
$$

Notice that since $l>0$, there is a natural mechanism forcing $\Phi$ to converge to zero at an exponential rate. However this fact necessitates modification of the arguments based on the lower bounds in lemma 3.2, which are dependent upon $\|\Phi\|_{L^{2}} \geq m>0$. The presence of $l>0$ in the integrals in the first and third identities means that for $\delta<\frac{l}{2}$ there exists $\tilde{\gamma}=\tilde{\gamma}\left(\mathcal{V}_{0}, l\right)$ such that

$$
\begin{gathered}
Q_{\mathbf{A}, \Phi}(\eta)+(l-\delta)\|\eta\|_{L^{2}}^{2} \geq \tilde{\gamma}\|\eta\|_{H_{\mathbf{A}}^{1}}^{2}, \quad \text { and } \\
\left\|\bar{\partial}_{\mathbf{A}} \Phi\right\|_{L^{2}}^{2}+(l-\delta)\|\Phi\|_{L^{2}}^{2} \geq \tilde{\gamma}\|\Phi\|_{H_{\mathbf{A}}^{1}}^{2} .
\end{gathered}
$$

For the middle identity, recall that if $\bar{y}=\frac{1}{|\Sigma|} \int_{\Sigma} y$ then Poincare's inequality says that

$$
\int e^{-2 \rho} \sum_{k}\left|\partial_{k} y\right|^{2} d \mu_{g} \geq C_{P}\|y-\bar{y}\|_{L^{2}}^{2} \geq C_{P}\left(\|y\|_{L^{2}}^{2}-\|\bar{y}\|_{L^{2}}^{2}\right),
$$

so that there exists $\gamma^{\prime}>0$ such that

$$
Q_{\Phi}(y) \geq \gamma^{\prime}\left(\|y\|_{H^{1}}^{2}-\|\bar{y}\|_{L^{2}}^{2}\right) .
$$

But also $l+\bar{y}=\frac{1}{|\Sigma|} \int_{\Sigma} v=l+\frac{1}{2|\Sigma|}\|\Phi\|_{L^{2}}^{2}$, so that $\bar{y}=\frac{1}{2|\Sigma|}\|\Phi\|_{L^{2}}^{2}$. Therefore, overall we have the following set of controlling inequalities:

$$
\begin{gathered}
e^{2 \delta T}\|\eta(T)\|_{L^{2}}^{2}+2 \int_{0}^{T} e^{2 \delta t} \tilde{\gamma}\|\eta\|_{H_{\mathbf{A}}^{1}}^{2} d t \leq\|\eta(0)\|_{L^{2}}^{2}-2 \int_{0}^{T} \int_{\Sigma} e^{2 \delta t} y|\eta|^{2} e^{-2 \rho} d \mu_{g} d t, \\
e^{2 \delta T}\|y(T)\|_{L^{2}}^{2}+2 \int_{0}^{T} e^{2 \delta t}\left(\left(\gamma^{\prime}-\delta\right)\|y\|_{H^{1}}^{2}-\gamma^{\prime}\|\bar{y}\|_{L^{2}}^{2}\right) d t \quad \leq\|y(0)\|_{L^{2}}^{2} \\
-2 \int_{0}^{T} \int_{\Sigma} e^{2 \delta t} y\left(|\Phi|^{2} l+4|\eta|^{2}\right) e^{-2 \rho} d \mu_{g} d t, \\
e^{2 \delta T}\|\Phi(T)\|_{L^{2}}^{2}+2 \int_{0}^{T} e^{2 \delta t} \tilde{\gamma}\|\Phi\|_{H_{\mathbf{A}}^{1}}^{2} d t \quad \leq\|\Phi(0)\|_{L^{2}}^{2}-2 \int_{0}^{T} \int_{\Sigma} e^{2 \delta t} y|\Phi|^{2} e^{-2 \rho} d \mu_{g} d t
\end{gathered}
$$

Add the three inequalities, and use the fact that $\|\bar{y}\|_{L^{2}}^{2}=|\Sigma||\bar{y}|^{2}=\frac{1}{2} \bar{y}\|\Phi\|_{L^{2}}^{2}$ to absorb the negative term in the integral in the second inequality by the integral in the third one. Next bound the nonlinear terms on the right hand sides in the same way as in (3.26)-(3.25), and conclude that as long as $\|y(0)\|_{L^{2}}$ is initially small then

$$
\begin{aligned}
& e^{2 \delta T}\|\eta(T)\|_{L^{2}}^{2} \leq\|\eta(0)\|_{L^{2}}^{2}, \\
& e^{2 \delta T}\|y(T)\|_{L^{2}}^{2} \leq\|y(0)\|_{L^{2}}^{2}, \\
& e^{2 \delta T}\|\Phi(T)\|_{L^{2}}^{2} \leq\|\Phi(0)\|_{L^{2}}^{2}
\end{aligned}
$$


holds for all $T>0$, and

$$
\int_{0}^{\infty} e^{2 \delta t}\left[\|y\|_{H^{1}}^{2}+\|\Phi\|_{H_{\mathbf{A}}^{1}}^{2}+\|\eta\|_{H_{\mathbf{A}}^{1}}^{2}\right] d t<\infty
$$

from which convergence can be deduced as in the previous section: (3.33) and (3.37) hold as a consequence of (3.49), and hence by the first two equations of (3.30) the estimate (3.41) also holds, and so $\mathbf{A}(t)$ converges to a limit $\mathbf{A}_{\infty}$ at an exponential rate. The proof of the main theorem is now complete.

\section{References}

[1] E. Bogomolny, Stability of Classical Solutions, Soviet Journal of Nulclear Physics 24 861-870 (1976).

[2] S. Bradlow, Vortices in holomorphic line bundles and closed Kaehler manifolds, Commun. Math. Phys. 118 1-17 (1990).

[3] S. Demoulini and D. Stuart, Adiabatic Limit and the Slow Motion of Vortices in a Chern-SimonsSchroedinger System, Commun. Math. Phys. 290, 597-632 (2009).

[4] S. Demoulini and D. Stuart, Gradient flow of the superconducting Ginzburg-Landau functional on the plane, Commun. Anal. Geom. 5(1) 121 - 198 (1997).

[5] S. Demoulini, Global existence for a nonlinear Schrödinger-Chern-Simons system on a surface, Ann. Inst. H. Poincaré Anal. Non Linéaire 24(2) 207-225 (2007).

[6] E. Feireisl and P. Takac, Long-time stabilization of solutions to the Ginzburg-Landau equations of superconductivity Monatsh. Math. 133, 3 197-221 (2001).

[7] A. Hassell The Yang-Mills-Higgs heat flow on $\mathbf{R}^{3}$ J. Funct. Anal. 111 (2) 431-448 (1993).

[8] A. Jaffe and C. Taubes, Vortices and Monopoles, Birkhauser, Boston, 1982.

[9] J. Jost, Riemannian geometry and geometric analysis, Springer-Verlag 1988.

[10] M. Noguchi, Yang-Mills-Higgs theory on a compact Riemann surface J. Math. Phys. 28, 2343 (1987).

[11] L. Simon Asymptotics for a class of nonlinear evolution equations, with applications to geometric problems Ann. of Math. 118 (3) 525-571 (1983).

[12] M. Taylor, Partial Differential Equations I-III, Applied Mathematical Sciences, vol 117, Springer-Verlag 1996. 